\title{
Parallel MPEG-2 Encoder on ATM and Ethernet-Connected Workstations
}

\author{
Shahriar M. Akramullah ${ }^{1}$, Ishfaq Ahmad ${ }^{2}$, and Ming L. Liou ${ }^{1}$ \\ 1 Department of EEE, \\ 2 Department of Computer Science \\ Hong Kong University of Science \& Tech., Clear Water Bay, Kowloon, Hong Kong
}

\begin{abstract}
We present a software-based parallel MPEG-2 video encoder implemented on a cluster of workstations connected via an ATM switch and also via Ethernet. We exploit parallelism on a Group of Picture (GOP) basis such that each GOP of the video is encoded by a particular processor. We propose a scheme for efficient I/O and data distribution.
\end{abstract}

\section{Introduction}

Video compression can be hardware-based [1] or software-based [2]3. However, a software solution is more flexible, and thus allows algorithmic improvements. Nonetheless, very high computation requirements of video applications can often overwhelm a single-processor sequential computer [2]. Therefore, it is natural to exploit the enormous computing power offered by parallel computing systems. With their high performance-cost ratio and efficient communication hardware/software, clusters of workstations are the most suitable candidates for computation-intensive applications like video compression.

In this work, we explore the use of general-purpose workstations enabling other jobs to run while compressing video data. The environment is off-line, but the aim is to achieve maximum possible encoding rate beyond the real-time speed. We compare the performance of the encoder when the interconnection is via Ethernet with that when it is via an ATM switch. We propose a scheme for efficient $\mathrm{I} / \mathrm{O}$ and data distribution. This scheme provides fast data retrieval as well as efficient scheduling and matching of $\mathrm{I} / \mathrm{O}$ and compression rates such that the entire system operates in a highly balanced fashion without any bottlenecks.

\section{The Parallel Encoder}

In order to achieve maximum possible encoding rate, we keep all of the processors busy in reading the data, performing the encoding, and writing the coded bitstream by scheduling the disks, I/O processors, and computing processors. In other words, the processor waiting times are minimized.

In distributing data, an I/O processor reads $m$ batches of uncompressed video frames and sends one batch to each compute processor in the group. While the 
$m$ compute processors are encoding, the I/O processor reads the next $m$ batches and waits. As soon as a compute processor finishes the encoding of a batch it sends a request to the I/O processor, which sends the next batch to the requester. After serving all the requests, the I/O processor reads the next $m$ batches and waits for the requests. The compute processors save the compressed data into a buffer, and write it to the disk when the buffer is full.

\section{Experimental Results}

We use a video test sequence named football of CCIR-601 format $(720 \times 480)$ and SIF format $(360 \times 240)$ as input to our parallel encoder. For message-passing among the workstations in the cluster, which consists of 20 Sun Ultra-1 workstations, we use Message Passing Interface (MPI) environment.

Table 1 . The frame encoding rate for the football sequence.

\begin{tabular}{|c|c|c|c|c|c|c|c|}
\hline \multirow{2}{*}{ No. of Processors } & \multirow{2}{*}{ Group Size } & \multicolumn{3}{|c|}{$\begin{array}{c}\text { Frame/sec. } \\
\text { using ATM switch }\end{array}$} & \multicolumn{3}{c|}{$\begin{array}{c}\text { Frame/sec. } \\
\text { using Ethernet }\end{array}$} \\
\cline { 3 - 8 } & & \multicolumn{2}{|c|}{ SIF } & CCIR-601 & \multicolumn{2}{|c|}{ SIF } & CCIR-601 \\
\cline { 3 - 8 } & $B=3$ & $B=6$ & $B=1$ & $B=3$ & $B=6$ & $B=1$ \\
\hline \hline 4 & 3 & 5.32 & 5.41 & 1.05 & 5.19 & 5.28 & 0.90 \\
\hline 8 & 7 & 12.55 & 12.78 & 2.13 & 6.92 & 7.31 & 1.31 \\
\hline 12 & 11 & 19.38 & 20.15 & 4.20 & 6.98 & 7.64 & 1.84 \\
\hline 16 & 15 & 23.81 & 24.15 & 4.85 & 7.17 & 7.72 & 2.42 \\
\hline 20 & 19 & 24.20 & 25.66 & 5.11 & 7.33 & 7.57 & 2.77 \\
\hline
\end{tabular}

There is only one $\mathrm{I} / \mathrm{O}$ processor which reads the uncompressed data from only one disk and sends to 3, 7, 11, 15 and 19 compute processors respectively. For SIF, a batch size $(B)$ of 6 frames results in less overall waiting time and therefore yields better results than a smaller batch size. However, for CCIR-601, due to limitation of memory, only a batch of one frame is used. The cluster of workstations performs significantly better while connected via the ATM switch due to its higher bandwidth $(155 \mathrm{Mb} / \mathrm{s})$ compared to the Ethernet $(10 \mathrm{Mb} / \mathrm{s})$.

\section{Conclusion}

We have discussed a scheme for combined scheduling of I/O, disks and processors. We have achieved an encoding rate of 25.66 and 5.11 frames/sec. for SIF and CCIR-601 respectively, using only 20 workstations. However, for optimal performance, a careful balance is necessary in determining the batch and group sizes (depending on the I/O processor's memory), for which work is currently underway. This work was partially supported by HKTIIT grant 92/93.001. 


\section{References}

1. T. Akiyama et al., "MPEG2 Video Codec using Image Compression DSP", IEEE Tran. on Consumer Electronics, vol. 40, No. 3, Aug. 1994, pp. 466-472.

2. S. M. Akramullah, I. Ahmad and M. L. Liou, "Performance of a Software-Based MPEG-2 Video Encoder on Parallel and Distributed Systems", IEEE Tran. on Circuits and Systems for Video Technology, vol. 7, NO. 4, Aug. 1997, pp. 687-695.

3. K. Shen, L. A. Rowe, E. J. Delp, "A Spatial-Temporal Parallel Approach for Realtime MPEG Video Compression", Proc. of 1996 International Conf. on Parallel Processing, VOL. 2, 1996, pp. 100-107. 